\title{
Validation of the Publication of New Names and New Combinations Previously Effectively Published Outside the IJSB
}

\author{
List No. $59 \dagger$
}

The purpose of this announcement is to effect the valid publication of the following new names and new combinations under the procedure described previously [Int. J. Syst. Bacteriol. 27(3):iv, 1977]. Authors and other individuals wishing to have new names and/or combinations included in future lists should send the pertinent reprint or a photocopy thereof to the IJSB (c/o ASM) for confirmation that all of the other requirements for valid publication have been met. It should be noted that the date of valid publication of these new names and combinations is the date of publication of this list, not the date of the original publication of the names and combinations. The authors of the new names and combinations are as given below, and these authors' names will be included in the author index of the present issue and in the volume author index in this issue of the IJSB.

Inclusion of a name on these lists validates the name and thereby makes it available in bacteriological nomenclature. The inclusion of a name on this list is not to be construed as taxonomic acceptance of the taxon to which the name is applied. Indeed, some of these names may, in time, be shown to be synonyms, or the organisms may be transferred to another genus, thus necessitating the creation of a new combination.

\begin{tabular}{|c|c|c|c|c|}
\hline Name & Proposed as: & Author(s) (reference) & Priority ${ }^{a}$ & Nomenclatural type ${ }^{b}$ \\
\hline Acidimicrobium & New genus & Clark and Norris (2) & 4 & Acidimicrobium ferrooxidans \\
\hline Acidimicrobium ferrooxidans & New species & Clark and Norris (2) & 4 & Strain ICP (= DSM 10331) \\
\hline Aeromonas bestiarum & New species & Ali et al. (1) & 1 & $\begin{array}{l}\text { Strain CDC } 9533-76(=\text { ATCC } 51108= \\
\quad \text { CIP 7430) }\end{array}$ \\
\hline Bacillus dipsosauri & New species & Lawson et al. (6) & 7 & Strain DD1 (= NCFB 3027) \\
\hline Brevibacterium otitidis & New species & Pascual et al. (10) & 3 & Strain A37/73 (= NCFB 3053) \\
\hline Chlorobium tepidum & New species & Wahlund et al. (13) & 5 & Strain TLS (= ATCC 49652) \\
\hline $\begin{array}{l}\text { Desulfomicrobium escambiense corrig. } \\
\text { (Desulfomicrobium escambium } \\
\text { [sic]) }\end{array}$ & New species & Sharak Genthner et al. (11) & 8 & $\begin{array}{l}\text { Strain ESC } 1(=\text { ATCC } 51164= \\
\quad \text { DSM 10707) }\end{array}$ \\
\hline Halomonas canadensis & New species & Huval et al. (3) & 2 & NRCC 41227 (= ATCC 43984) \\
\hline Halomonas israelensis & New species & Huval et al. (3) & 2 & Strain $\mathrm{Ba}_{1}(=$ ATCC 43985) \\
\hline Heliobacterium gestii & New species & Ormerod et al. (9) & 5 & Strain Chainat $(=$ ATCC 43375) \\
\hline Heliobacterium modesticaldum & New species & Kimble et al. (5) & 5 & Strain Ice 1 (= ATCC 51547) \\
\hline Heliophilum & New genus & Ormerod et al. (9) & 5 & Heliophilum fasciatum \\
\hline Heliophilum fasciatum & New species & Ormerod et al. (9) & 5 & Strain Tanzania $(=$ ATCC 51790) \\
\hline Natroniella & New genus & Zhilina et al. (14) & 6 & Natroniella acetigena \\
\hline Natroniella acetigena & New species & Zhilina et al. (14) & 6 & Strain Z-7937 (= DSM 9952) \\
\hline Rhodospirillum sodomense & New species & Mack et al. (7) & 5 & Strain DSI (= ATCC 51195) \\
\hline $\begin{array}{l}\text { Saccharothrix aerocolonigenes subsp. } \\
\text { aerocolonigenes }\end{array}$ & Rule $46^{c}$ & & & \\
\hline $\begin{array}{l}\text { Saccharothrix aerocolonigenes subsp. } \\
\text { staurosporeus (basonym: "Strepto- } \\
\text { myces stauroporeus") }\end{array}$ & $\begin{array}{l}\text { New subspecies, new combi- } \\
\text { nation, revived name }\end{array}$ & Takahashi et al. (12) & 3 & Strain AM-2282 (= NRRL 11184) \\
\hline $\begin{array}{l}\text { Streptosporangium amethystogenes } \\
\text { subsp. amethystogenes }\end{array}$ & Rule $46^{d}$ & & & \\
\hline $\begin{array}{l}\text { Streptosporangium amethystogenes } \\
\text { subsp. fukuiense }\end{array}$ & New subspecies & Iinuma et al. (4) & 9 & Strain AL-23456 (= IFO 15365) \\
\hline Sulfobacillus acidophilus & New species & Norris et al. (8) & 4 & Strain NAL (= DSM 10332) \\
\hline
\end{tabular}

$\dagger$ Lists 1 through 58 were published in the Int. J. Syst. Bacteriol. 27:306, 1977; 29:79, 436, 1979; 30:601, 676, 1980; 31:215, 382, 1981; 32:266, 384, 1982; 33:438, 672, 896,$1983 ; \mathbf{3 4}: 91,270,355,503,1984 ; \mathbf{3 5}: 223,375,535,1985 ; \mathbf{3 6}: 354,489,573,1986 ; \mathbf{3 7}: 179,1987 ; \mathbf{3 8}: 136,220,328,449,1988 ; \mathbf{3 9}: 93,205,371,495,1989 ; \mathbf{4 0}: 105,212$, $320,470,1990 ; \mathbf{4 1}: 178,331,456,580,1991 ; 42: 191,327,511,656,1992 ; 43: 188,398,624,864,1993 ; 44: 182,370,595,852,1994 ; 45: 197,418,619,879,1995 ;$ and 46:362, $625,836,1996$.

a Priority number assigned according to the date the documentation and request for validation are received.

${ }^{b}$ Abbreviations: ATCC, American Type Culture Collection, Rockville, Md.; CIP, Collection of the Institute Pasteur, Paris, France; DSM, DSMZ-Deutsche Sammlung von Mikroorganismen und Zellkulturen GmbH, Braunschweig, Germany; IFO, Institute for Fermentation, Osaka, Japan; NCFB, National Collection of Food Bacteria, Institute of Food Research, Reading, England; NRCC, National Research Council of Canada, Ottawa, Ontario, Canada; NRRL, Agricultural Research Service Culture Collection, National Center for Agricultural Utilization Research, U.S. Department of Agriculture, Peoria, Ill.

cAccording to Rule 46 of the International Code of Nomenclature of Bacteria, the valid publication of Saccharothrix aerocolonigenes subsp. staurosporeus auto matically creates another subspecies Saccharothrix aerocolonigenes subsp. aerocolonigenes Labeda 1986.

${ }^{d}$ According to Rule 46 of the International Code of Nomenclature of Bacteria, the valid publication of Streptosporangium amethystogenes subsp. fukuiense automatically creates another subspecies Streptosporangium amethystogenes subsp. amethystogenes Nonomura and Ohara 1960. 


\section{REFERENCES}

1. Ali, A., A. M. Carnahan, M. Altwegg, J. Lüthy-Hottenstein, and S. W. Joseph. 1996. Aeromonas bestiarum sp. nov. (formerly genomospecies DNA group $2 A$. hydrophila), a new species isolated from non-human sources. Med. Microbiol. Lett. 5:156-165.

2. Clark, D. A., and P. R. Norris. 1996. Acidimicrobium ferrooxidans gen. nov., sp. nov.: mixed-culture ferrous iron oxidation with Sulfobacillus species. Microbiology 142:785-790.

3. Huval, J. H., R. Latta, R. Wallace, D. J. Kushner, and R. H. Vreeland. 1995. Description of two new species of Halomonas: Halomonas israelensis sp. nov. and Halomonas canadensis sp. nov. Can. J. Microbiol. 41:1124-1131.

4. Iinuma, S., A. Yokota, and T. Kanamura. 1996. New subspecies of the genus Streptosporangium, Streptosporangium amethystogenes subsp. fukuiense subsp. nov. Actinomycetologica 10:35-42.

5. Kimble, L. K., L. Mandelco, C. R. Woese, and M. T. Madigan. 1995. Heliobacterium modesticaldum, sp. nov., a thermophilic heliobacterium of hot springs and volcanic soils. Arch. Microbiol. 163:259-267.

6. Lawson, P. A., C. E. Deutsch, and M. D. Collins. 1996. Phylogenetic characterization of a novel salt-tolerant Bacillus species: description of Bacillus dipsosauri sp. nov. J. Appl. Bacteriol. 81:109-112.

7. Mack, E. E., L. Mandelco, C. R. Woese, and M. T. Madigan. 1993. Rhodospirillum sodomense, sp. nov., a Dead Sea Rhodospirillum species. Arch. Microbiol. 160:363-371.

8. Norris, P. R., D. A. Clark, J. P. Owen, and S. Waterhouse, 1996. Characteristics of Sulfobacillus acidophilus sp. nov. and other moderately thermophilic mineral-sulphide-oxidizing bacteria. Microbiology 142:775-783.

9. Ormerod, J. G., L. K. Kimble, T. Nesbakken, Y. A. Torgersen, C. R. Woese, and M. T. Madigan. 1996. Heliophilum fasciatum gen. nov. sp. nov. and Heliobacterium gestii sp. nov.: endospore-forming heliobacteria from rice field soils. Arch. Microbiol. 165:226-267.

10. Pascual, C., M. D. Collins, G. Funke, and D. G. Pitcher. 1996. Phenotypic and genotypic characterization of two Brevibacterium strains from the human ear: description of Brevibacterium otitidis sp. nov. Med. Microbiol. Lett. 5:113-123.

11. Sharak Genthner, B. R., G. Mundfrom, and R. Devereux. 1994. Characterization of Desulfomicrobium escambium sp. nov. and proposal to assign Desulfovibrio desulfuricans Norway 4 to the genus Desulfomicrobium. Arch. Microbiol. 161:215-219.

12. Takahashi, Y., M. Shinose, A. Seino, Y. Iwai, and S. Omura. 1995. Transfer of staurosporine-producing strain Streptomyces staurosporeus AM-2282 to the genus Saccharothrix as Saccharothrix aerocolonigenes (Labeda 1986) subsp. staurosporeus subsp. nov. Actinomycetologica 9:19-26.

13. Wahlund, T. M., C. R. Woese, R. W. Castenholz, and M. T. Madigan. 1991. A thermophilic green sulfur bacterium from New Zealand hot springs, Chlorobium tepidum sp. nov. Arch. Microbiol. 156:81-90.

14. Zhilina, T. N., G. A. Zavarzin, E. N. Detkova, and F. A. Rainey. 1996. Natroniella acetigena gen. nov. sp. nov., an extremely haloalkaliphilic, homoacetic bacterium: a new member of Haloanaerobiales. Curr. Microbiol. 32:320-326. 